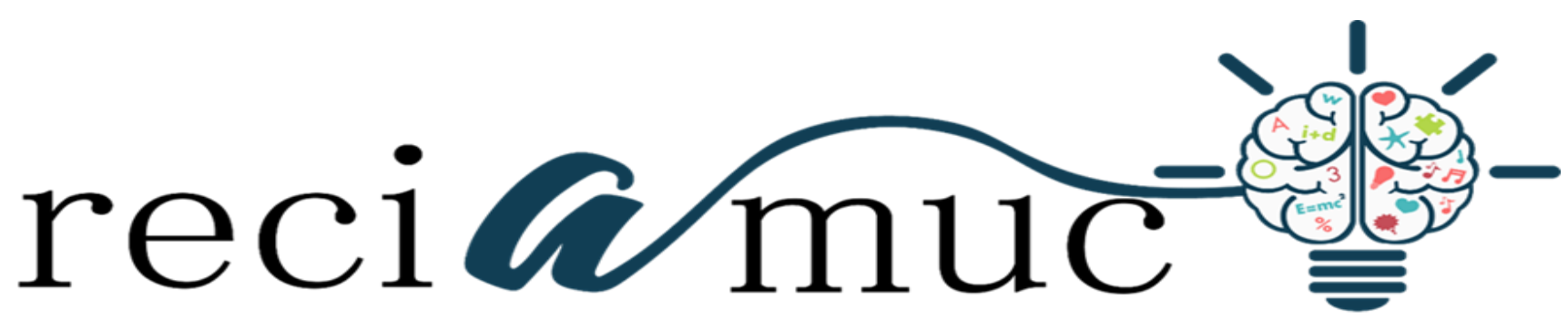

Revista científica de investigación actualización del mundo de las ciencias

Leurys Beatriz Moreira Plaza a; Viviana del Rocío Moreira Quiroz ${ }^{\text {b; }}$ Adriana Carolina Romero Vega ${ }^{c}$; Mayteé Celina Guerrero Cabrera ${ }^{d}$; Fabián Xavier Moreira Rivadeneira ${ }^{\mathrm{e}}$; Giancarlo Cristóbal Cañarte Baque ${ }^{\mathrm{f}}$

Prevención y cuidados ginecológicos de la mujer en edad fértil Prevention and gynecological care of women of fertile age

Revista Científica de Investigación actualización del mundo de las Ciencias. Vol. 3 núm., 3, julio, ISSN: 2588-0748, 2018, pp. 1303-1322

DOI: 10.26820/reciamuc/3.(3).julio.2019.1303-1322

URL: http://reciamuc.com/index.php/RECIAMUC/article/view/383

Código UNESCO: 3205 Medicina Interna

Tipo de Investigación: Artículo de Investigación

() RECIAMUC; Editorial Saberes del Conocimiento, 2019

Recibido: 28/04/2019

Aceptado: 19/05/2019

Publicado: 01/07/2019

Correspondencia: leurismp@ hotmail.com
a. Médico General Asistencial - Hospital Verdi Cevallos; Portoviejo - Ecuador; leurismp @ hotmail.com
b. Médico General Asistencial - Hospital Verdi Cevallos; Portoviejo - Ecuador; vivirociomoreira@ gmail.com
c. Médico Residente Hospital Clínica Kennedy; Guayaquil - Ecuador; adri_romero2112@ hotmail.com
d. Médica; Investigador Independiente; Cuenca - Ecuador; mayt_cel@ hotmail.com
e. Médico Cirujano; Investigador Independiente; Manta - Ecuador; famori82@ gmail.com
f. Médico General Asistencial Cirugía General; Hospital Verdi Cevallos Balda; Portoviejo - Ecuador; gcanarte@gmail.com 


\section{Prevención y cuidados ginecológicos de la mujer en edad fértil}

Vol. 3, núm. 3., (2019)

Leurys Beatriz Moreira Plaza; Viviana del Rocío Moreira Quiroz; Adriana Carolina Romero

Vega; Mayteé Celina Guerrero Cabrera; Fabián Xavier Moreira Rivadeneira; Giancarlo Cristóbal Cañarte Baque

\section{RESUMEN}

Las mujeres en edad fértil presentan una serie de características que definen no solo su salud y bienestar, sino que también se convierten en factores positivos o negativos predictores de la salud de las futuras generaciones. Estas féminas en edades entre los 15 a los 49 años de edad representan, sin lugar a dudas un grupo etario fundamental en el logro de la salud global, lo que lleva a hacer énfasis en el logro de una buena etapa pre-concepcional femenina. De ahí, la importancia que tienen los cuidados ginecológicos, entendidos como un instrumento básico indispensable para prevenir o detectar numerosas patologías femeninas, este tipo de intervenciones médicas forman parte fundamental en la exploración clínica y permiten orientar hacia un posible diagnóstico, desarrollado, a partir de la actividad sexual de la mujer y aplicado cada año de manera periódica. La puesta en práctica de estos cuidados ginecológicos deben contemplar inicialmente, un interrogatorio que permita determinar los motivos de consulta, investigar los antecedentes familiares, los síntomas y la actividad sexual de la paciente, a fin de llevar a cabo los respectivos exámenes de rutina y los relacionados a descartar cualquiera anomalía o patología oncológica en los órganos sexuales y las mamas, como por ejemplo el cáncer (en sus diferentes presentaciones o tipos), el VIH, Papilomas, entre otras; planteamientos éstos que llevan a establecer que la evaluación y cuidados ginecológicos de la mujer en edad fértil debe ser integral y desarrollado de forma periódica, los mismos deben percibirse como una estrategia complementaria en la lucha por disminuir la mortalidad femenina del país, ya que a través de este tipo de revisiones médicas pueden ser descubiertas precozmente una serie de patologías, entre ellas las de tipo oncológico aumentando las posibilidades de curación, sobre todo en aquellos casos, como son los procesos neoplásicos o tumores.

Palabras Claves: Prevención; Cuidados ginecológicos; Edad Fértil; Cáncer; VIH; Virus de Papiloma; Patologías Femeninas. 


\title{
Prevención y cuidados ginecológicos de la mujer en edad fértil
}

Vol. 3, núm. 3., (2019)

Leurys Beatriz Moreira Plaza; Viviana del Rocío Moreira Quiroz; Adriana Carolina Romero Vega; Mayteé Celina Guerrero Cabrera; Fabián Xavier Moreira Rivadeneira; Giancarlo Cristóbal

Cañarte Baque

\begin{abstract}
Women of childbearing age have a series of characteristics that define not only their health and well-being, but also become positive or negative predictors of the health of future generations. These females between the ages of 15 and 49 represent, without a doubt, a fundamental age group in the achievement of global health, which leads to an emphasis on the achievement of a good preconceptional stage of women. Hence, the importance of gynecological care, understood as an essential basic instrument to prevent or detect numerous female pathologies, this type of medical interventions is a fundamental part of the clinical examination and allow us to orient towards a possible diagnosis, developed, based on the sexual activity of women and applied every year periodically. The implementation of these gynecological care should initially include an interrogation that allows to determine the reasons for consultation, investigate the family history, symptoms and sexual activity of the patient, in order to carry out the respective routine examinations and related to ruling out any abnormality or oncological pathology in the sexual organs and breasts, such as cancer (in its different presentations or types), HIV, Papillomas, among others; These approaches lead to establish that the evaluation and gynecological care of women of childbearing age must be comprehensive and developed periodically, they should be perceived as a complementary strategy in the fight to reduce female mortality in the country, since through From this type of medical check-ups, a series of pathologies can be discovered early, including those of an oncological type, increasing the chances of cure, especially in those cases, such as neoplastic processes or tumors.
\end{abstract}

Key Words: Prevention; Gynecological care; Fertile age; Cancer; HIV; Papillomavirus; Female Pathologies. 


\section{Prevención y cuidados ginecológicos de la mujer en edad fértil}

Vol. 3, núm. 3., (2019)

Leurys Beatriz Moreira Plaza; Viviana del Rocío Moreira Quiroz; Adriana Carolina Romero Vega; Mayteé Celina Guerrero Cabrera; Fabián Xavier Moreira Rivadeneira; Giancarlo Cristóbal Cañarte Baque

\section{Introducción.}

Para muchas mujeres, el estudio ginecólogo representa la oportunidad de prevenir y tratar gran variedad de enfermedades, tomando en cuenta que, la frecuencia de estas patologías, va a variar según el grupo de edad que la fémina presente: de allí, que cada consulta ginecológica debe tomar en cuenta las necesidades propias de la edad de cada paciente y estar orientada a preparar a la mujer para cada época, buscándose con ello que la misma logre identificar tempranamente cualquier alteración que llegase a presentar tratándolas de la manera más oportuna e integral cuando éstas ya están presentes. (Cett, 2016).

Si bien la edad para iniciar los exámenes ginecológicos no ha sido definida, lo más conveniente es que sea realizada la primera a los (14 ó 15 años), buscándose con ello, que la adolescente logre plantearle al médico tratante todas sus dudas, lográndose con ello que la misma conozca sobre métodos anticonceptivos y enfermedades vinculadas a las zonas reproductivas. Esta primera consulta, es conceptualizada médicamente como la oportunidad de estas jóvenes de "romper el hielo", lo que les permite a estas féminas en primeras edades comprender la importancia que tiene el asistir de manera periódica al ginecólogo.

De esta manera, el chequeo ginecológico durante la edad reproductiva constituirá uno de los procesos esenciales y de rutina en la vida reproductiva de la mujer, permitiéndole a la misma identificar de manera temprana los factores de riesgo que pudieran incidir sobre la aparición de alguna enfermedad y tener conciencia plena sobre los métodos anticonceptivos existentes y la importancia e importancia que tiene un embarazo deseado. Para (Perroni, 2016), “el examen ginecológico constituye un instrumento básico como medio de prevención o detección precoz, que forma parte de un importante paso en la exploración clínica y orienta hacia un probable diagnóstico". (p.54).

Por consiguiente, se puede decir que entre los cuidados ginecológicos que debe seguir la mujer en edad fértil y todas las etapas de su vida, se encuentran los referidos a la atención 


\section{Prevención y cuidados ginecológicos de la mujer en edad fértil}

Vol. 3, núm. 3., (2019)

Leurys Beatriz Moreira Plaza; Viviana del Rocío Moreira Quiroz; Adriana Carolina Romero Vega; Mayteé Celina Guerrero Cabrera; Fabián Xavier Moreira Rivadeneira; Giancarlo Cristóbal

Cañarte Baque

preventiva, es decir, a todos aquellos programas y acciones de salud que contemplen y detección precoz de enfermedades de mayor incidencia que afectan a la mujer, los cuales permitan chequear el estado de la salud de la misma, facilitando el hallazgo precoz de ciertas afecciones médicas, incrementando las posibilidades de actuar oportunamente ante patologías, como: el cáncer de mama y cuello uterino, el cáncer de ovario, papiloma humano, entre otras de orden crónico.

Dicho de esta forma, es de gran importancia que la mujer se realice estudios ginecológicos de orden preventivo; en especial, las mujeres en edad fértil o reproductiva las cuales tienden a presentar una serie de patologías que ponen en riesgo su salud y calidad de vida, llegando incluso a ocasionarles hasta la muerte. Entre ellas destacan las afecciones ginecológicas, reconocidas como una de las más frecuentes a la que se ven expuestas las mujeres en edad reproducida (15 a 44 años), las cuales derivan del cáncer genitourinario y de mama. Asimismo, se presenta el VIH que constituye la principal causa de mortalidad a nivel mundial, mientras que las relaciones sexuales no seguras. (Rawy, 2015).

En pocas palabras, es obligatorio que la mujer chequee de forma periódica su estado de salud ginecológico, ya que existen enfermedades que pueden ser descubiertas precozmente aumentando las posibilidades de curación como son los procesos neoplásicos o tumores. Estas revisiones del estado de salud, deben ser adaptadas a cada persona según antecedentes familiares, personales y estilo de vida, tomando en cuenta que, los factores biológicos, la falta de acceso a la información, los servicios de salud, la vulnerabilidad económica y la desigualdad de poder en las relaciones sexuales exponen a las mujeres, en particular a las jóvenes, al riesgo de infección por el VIH, Papiloma Humano, Sífilis, Gonorrea, entre otras afecciones de igual riesgo.

De igual manera, se debe señalar la tuberculosis; enfermedad ésta que de manera regular es relacionada con la infección por el VIH, sobre todo, en los países de ingresos bajos, por cuanto, en ellos, esta patología en mujeres en edad fértil se encuentra ubicada 


\section{Prevención y cuidados ginecológicos de la mujer en edad fértil}

Vol. 3, núm. 3., (2019)

Leurys Beatriz Moreira Plaza; Viviana del Rocío Moreira Quiroz; Adriana Carolina Romero Vega; Mayteé Celina Guerrero Cabrera; Fabián Xavier Moreira Rivadeneira; Giancarlo Cristóbal Cañarte Baque

epidemiológicamente entre las cinco causas de defunción. Por otra parte, estadísticas mundiales hacen referencia al caso de las defunciones maternas, las cuales ocupan el segundo lugar dentro de las causas de mortalidad que afectan a las mujeres en edad fecunda, cada una de ellas, fallecidas a causas de complicaciones generadas por el embarazo o el parto, lo que se ve reflejado en unas 287000 féminas fallecidas, el 99\% de ellas en países en desarrollo.

En efecto, es de gran valor entender que los cuidados ginecológicos representan uno de los hechos más importantes en la medicina preventiva; los mismos y los cuidados y preparación educativa que brindan los mismos a las mujeres en edad fértil, contribuyen de gran forma, a minimizar los riesgos a contraer enfermedades de transmisión sexual y patologías como el papiloma, cáncer y sus diferentes presentaciones, flagelo éste que en muchos de los casos de debe, al profundo desconocimiento que estas enfermedades tienen acerca de las mismas, sus riesgos y sus consecuencias. De aquí, la importancia de promover campañas que brinden informaciones de salud a estas poblaciones, las cuales orientadas de forma efectiva le permitirán adquirir, aprender y tomar conciencia sobre los beneficios que brinda el desarrollo un cuidado ginecológico y una educación sexual responsable y segura.

De allí, la importancia de fomentar el desarrollo de planes en atención primaria y charlas educativas liderizadas, a través de diversas organizaciones encargadas de emitir normas para los cuidados profilácticos. Estas organizaciones comprenden a los Centers for Disease Control and Prevention (CDC), el U.S. Preventive Services Task Force (USPSTF), la American Cancer Society y el American College of Obstetricians and Gynecologists que le permitan a la mujer en sus diferentes etapas de la vida desarrollar cuidados responsable; ya que, si no se educa en la sexualidad y los riesgos biológicos y patológicos a los que se enfrenta la mujer en determinadas edades, se fomenta la ignorancia y el riesgo de adquirir este tipo enfermedades.

Tales organizaciones dirigen su atención preventiva en el desarrollo de programas que involucren la educación y orientación de temáticas de orden sanitario que permitan a la mujer alcanzar niveles de vida óptimos; entre ellos se destacan: 


\section{Prevención y cuidados ginecológicos de la mujer en edad fértil}

Vol. 3, núm. 3., (2019)

Leurys Beatriz Moreira Plaza; Viviana del Rocío Moreira Quiroz; Adriana Carolina Romero Vega; Mayteé Celina Guerrero Cabrera; Fabián Xavier Moreira Rivadeneira; Giancarlo Cristóbal Cañarte Baque

Cuadro $\mathrm{N}^{\circ}$ 1. Actuaciones que manejan los Programas de Atención Primaria dirigidos a la Mujer

\begin{tabular}{|ll|}
\hline Edad & Programa \\
\hline Mujeres de 15 a 34 años & Información y Seguimiento de Anticonceptivos \\
\hline Mujeres de 35 a 49 años & $\begin{array}{l}\text { Información y Seguimiento de Anticonceptivos } \\
\text { Detección Precoz de Cáncer de Cervix }\end{array}$ \\
Mujeres de 50 a 64 años & $\begin{array}{l}\text { Detección Precoz de Cáncer de Mama } \\
\text { Detección Precoz de Cáncer de Endometrio } \\
\text { Detección Precoz de Cáncer de Cervix } \\
\text { Atención al Climaterio }\end{array}$ \\
\hline
\end{tabular}

Fuente: Organización Mundial de la Salud OMS (2017).

Por ende, la formulación y puesta en práctica de estos Planes de Atención Primaria ejecutan e implementan orientaciones sanitarias dirigidas a orientar a las féminas sobre temáticas que involucren:

- Situación actual del proceso/enfermedad

- Atención actual que presta el INSALUD

- Objetivos

- Población diana (referida a la base de datos de tarjeta sanitaria

- individual)

- Actuaciones

- Organización y Gestión

- Costes (referidos a los dos primeros años)

- Evaluación. 


\section{Prevención y cuidados ginecológicos de la mujer en edad fértil}

Vol. 3, núm. 3., (2019)

Leurys Beatriz Moreira Plaza; Viviana del Rocío Moreira Quiroz; Adriana Carolina Romero

Vega; Mayteé Celina Guerrero Cabrera; Fabián Xavier Moreira Rivadeneira; Giancarlo Cristóbal Cañarte Baque

\section{Tipo de Investigación.}

Dentro de toda práctica investigativa, se precisan acciones de carácter metodológico mediante las cuales, se logra conocer y proyectar los eventos posibles que la determinan, así como las características que hacen del acto científico un proceso interactivo ajustado a una realidad posible de ser interpretada. En este sentido, se puede decir, que la presente investigación corresponde al tipo documental, definido por (Dávila, 2012), "se ocupa del estudio de problemas planteados a nivel teórico, la información requerida para abordarlos se encuentra básicamente en materiales impresos, audiovisuales y /o electrónicos”. (p.41).

En consideración a esta definición, la orientación metodológica permitió la oportunidad de cumplir con una serie de actividades inherentes a la revisión y lectura de diversos documentos donde se encontraron ideas explicitas relacionadas con los tópicos encargados de identificar a cada característica insertada en el estudio. Por lo tanto, se realizaron continuas interpretaciones con el claro propósito de revisar aquellas apreciaciones o investigaciones propuestas por diferentes investigadores, para luego dar la respectiva argumentación a los planteamientos, en función a las necesidades encontradas en la indagación.

\section{Fuentes Documentales.}

El análisis correspondiente a las características que predomina en el tema seleccionado, llevan a incluir diferentes fuentes documentales encargadas de darle el respectivo apoyo y en ese sentido cumplir con la valoración de los hechos a fin de generar nuevos criterios que sirven de referencia a otros procesos investigativos. Para (Arias, 2010), las fuentes documentales incorporadas en la investigación documental o bibliográfica, "representa la suma de materiales sistemáticos que son revisados en forma rigurosa y profunda para llegar a un análisis del fenómeno”. (p.41). Por lo tanto, se procedió a cumplir con la realización de una lectura previa determinada por encontrar aquellos aspectos estrechamente vinculados con la "Prevención y cuidados ginecológicos de la mujer en edad Fértil" para luego explicar mediante un desarrollo las respectivas apreciaciones generales de importancia. 


\section{Prevención y cuidados ginecológicos de la mujer en edad fértil}

Vol. 3, núm. 3., (2019)

Leurys Beatriz Moreira Plaza; Viviana del Rocío Moreira Quiroz; Adriana Carolina Romero Vega; Mayteé Celina Guerrero Cabrera; Fabián Xavier Moreira Rivadeneira; Giancarlo Cristóbal

Cañarte Baque

\section{Técnicas para la Recolección de la Información.}

La conducción de la investigación para ser realizada en función a las particularidades que determinan a los estudios documentales, tiene como fin el desarrollo de un conjunto de acciones encargadas de llevar a la selección de técnicas estrechamente vinculadas con las características del estudio. En tal sentido, (Arias Ob cit) refiere, que es "una técnica particular para aportar ayuda a los procedimientos de selección de las ideas primarias y secundarias”. (p. 71).

Por ello, se procedió a la utilización del subrayado, resúmenes, fichaje, como parte básica para la revisión y selección de los documentos que presentan el contenido teórico. Es decir, que mediante su aplicación de estas técnicas se pudo llegar a recoger informaciones en cuanto a la revisión bibliográfica de los diversos elementos encargados de orientar el proceso de investigación. Tal como lo expresa, (Arias $\mathrm{Ob}$ cit) "las técnicas documentales proporcionan las herramientas esenciales y determinantes para responder a los objetivos formulados y llegar a resultados efectivos" (p. 58). Es decir, para responder con eficiencia a las necesidades investigativas, se introdujeron como técnica de recolección el método inductivo, que hizo posible llevar a cabo una valoración de los hechos de forma particular para llegar a la explicación desde una visión general.

Asimismo, se emplearon las técnicas de análisis de información para la realización de la investigación que fue ejecutada bajo la dinámica de aplicar diversos elementos encargados de determinar el camino a recorrer por el estudio, según, (Arias, Ob cit) las técnicas de procesamiento de datos en los estudios documentales "son las encargadas de ofrecer al investigador la visión o pasos que debe cumplir durante su ejercicio, cada una de ellas debe estar en correspondencia con el nivel a emplear" (p. 123). Esto indica, que para llevar a cabo el procesamiento de los datos obtenidos, es necesario establecer las técnicas que serán seleccionadas, destacándose en este caso, de manera particular: fichas de resumen, textual, registros descriptivos entre otros, los mismos se deben ajustar al nivel que ha sido seleccionado.

\section{Resultados.}

Cuidados Ginecológicos de la Mujer en Edad Fértil. 


\section{Prevención y cuidados ginecológicos de la mujer en edad fértil}

Vol. 3, núm. 3., (2019)

Leurys Beatriz Moreira Plaza; Viviana del Rocío Moreira Quiroz; Adriana Carolina Romero

Vega; Mayteé Celina Guerrero Cabrera; Fabián Xavier Moreira Rivadeneira; Giancarlo Cristóbal Cañarte Baque

La salud es el estado de completo bienestar físico, mental y social y no solamente la ausencia de enfermedad; La salud de las mujeres se refiere a la rama de la medicina que se enfoca en el tratamiento y diagnóstico de enfermedades y padecimientos que afectan el bienestar físico y emocional de la misma. Según (Riantt, 2017), “el control ginecológico preventivo representa una de las actividades pertenecientes a la atención primaria, como a la atención de salud ginecológica integral realizada a la población femenina a lo largo de su ciclo vital. (p.32). En efecto, el control ginecológico representa una de las acciones en salud de gran importancia sanitaria; entre sus propósitos se encuentra:

- Detectar precozmente trastornos de la nutrición.

- Prevenir y pesquisar exposiciones a riesgos laborales y ambientales.

- Prevenir y detectar precozmente afecciones ginecológicas.

- Promover la detección de las enfermedades de transmisión sexual y SIDA.

- Prevenir y pesquisar factores de riesgo para patología cardiovascular, obesidad, hipertensión, diabetes, cánceres (cervico uterino, mama, vesicular).

- Pesquisar y detectar enfermedades crónicas en las diferentes etapas del ciclo vital de la mujer.

- Pesquisar morbilidad que pueda afectar a la mujer en el periodo climatérico y senescencia.

- Explorar aspectos de violencia intrafamiliar.

- Realizar adecuado seguimiento de la población femenina beneficiaria.

En términos generales, el cuidado o control ginecológico preventivo es aquel que permite a la mujer dar pasos simples para prevenir infectarse o propagar el VIH/SIDA y otras infecciones que se transmiten sexualmente (ITS), las cuales requieren tomar el control y recibir tratamiento. De igual forma, este cuidado, no sólo es importante para impedir que se propague la infección, sino para recibir tratamiento, lo que en su efecto permitiría detener graves problemas, entre ellos la esterilidad e incluso la muerte.

En efecto, datos epidemiológicos demuestran que, en el grupo de 35 a 64 años de mujeres, las tres primeras causas de mortalidad son los tumores malignos (siendo los más comunes lo del área ginecológica), las enfermedades del corazón y las cerebrovasculares. La cuarta causa la reflejan, 


\section{Prevención y cuidados ginecológicos de la mujer en edad fértil}

Vol. 3, núm. 3., (2019)

Leurys Beatriz Moreira Plaza; Viviana del Rocío Moreira Quiroz; Adriana Carolina Romero Vega; Mayteé Celina Guerrero Cabrera; Fabián Xavier Moreira Rivadeneira; Giancarlo Cristóbal

Cañarte Baque

los accidentes y efectos adversos y quinta la septicemia, para las mujeres de 50 años y más comienza a ocupar este lugar la diabetes mellitus. (Calls, 2016).

En base a Estos señalamientos, (Pallos, 2016), expresa existen programas de orden ginecológicos destinados a conservar la salud de la mujer que tienen como propósito:

- Contribuir a mejorar las condiciones de salud de las mujeres. La estrategia para alcanzar este objetivo está centrada en la promoción de la participación activa y solidaria de la mujer, a través de organizaciones de la comunidad, en la gestión local para obtener una mejor calidad de vida para sí, para su familia y para su comunidad.

- Contribuir a mejorar la atención y los servicios de salud teniendo en cuenta las necesidades de las mujeres en los distintos períodos del ciclo vital: niñez, adolescencia, edad adulta, edad mediana y vejez. El enfoque estratégico del programa para alcanzar este objetivo es el de trabajar coordinadamente con otros programas del Ministerio, partiendo del supuesto de que al transversalizar la problemática de género en la investigación y capacitación del recurso humano para la atención, prevención y promoción de la salud de la mujer estaremos contribuyendo a su desarrollo integral. 


\section{Prevención y cuidados ginecológicos de la mujer en edad fértil}

Vol. 3, núm. 3., (2019)

Leurys Beatriz Moreira Plaza; Viviana del Rocío Moreira Quiroz; Adriana Carolina Romero Vega; Mayteé Celina Guerrero Cabrera; Fabián Xavier Moreira Rivadeneira; Giancarlo Cristóbal Cañarte Baque

\section{Imagen $\mathbf{N}^{\circ}$ 1. Corte transversal Útero}

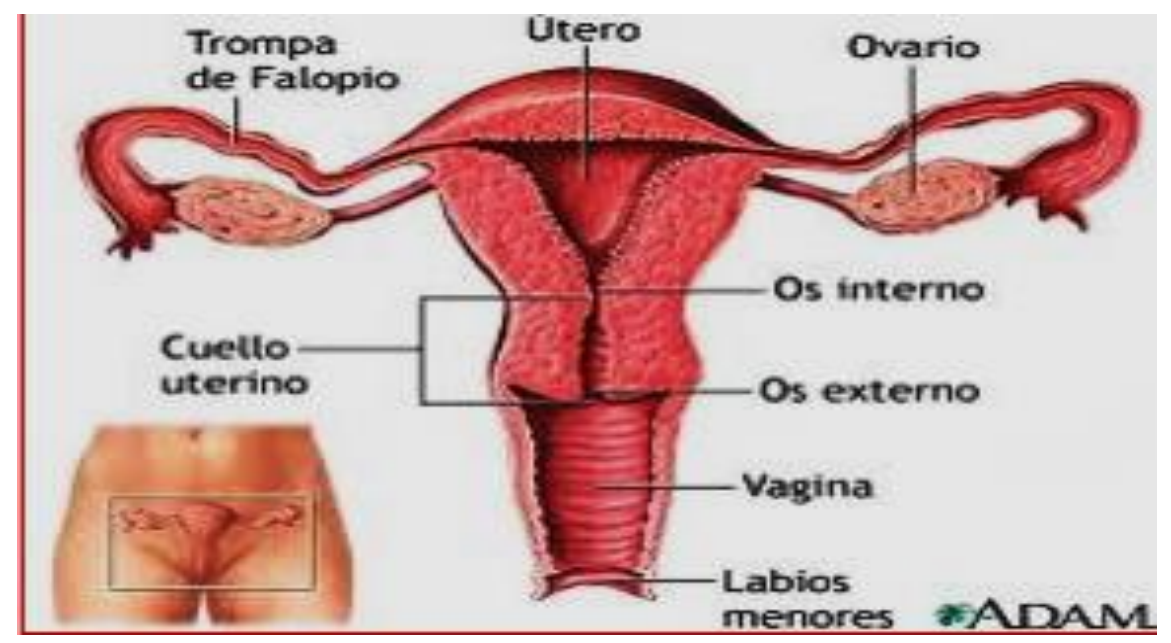

Fuente: (Calls 2016).

- Salud reproductiva es, según la Organización Mundial de la Salud OMS, el estado de completo bienestar físico, mental y social, en los aspectos relativos a la sexualidad y la reproducción en todas las etapas de la vida. No es por tanto una mera ausencia de enfermedades dolencias. La salud reproductiva implica que las personas puedan tener una vida sexual y segura y satisfactoria, la capacidad de tener hijos y la libertad de decidir si quieren tenerlos, cuando y con qué frecuencia. Tanto el hombre como la mujer tienen derecho a estar informados y tener acceso a métodos de regulación de la fertilidad seguros, eficaces y asequibles, así como a disponer de servicios accesibles de planificación familiar y salud pública que permitan la adecuada asistencia profesional a la mujer embarazada y permitan que el parto se produzca de forma segura y garantice el nacimiento de hijos sanos. (p.21).

Entre las Patologías más Comunes que pueden ser identificadas y orientadas en la Mujer en Edades Fértiles se encuentran:

El cáncer de endometrio es el segundo en orden de frecuencia de los cánceres ginecológicos, después del de mama. Ocupa el primer lugar de los cánceres uterinos, por delante del cáncer de cuello, si se excluye la neoplasia cervical intra epitelial, afectando de forma casi exclusiva a 


\section{Prevención y cuidados ginecológicos de la mujer en edad fértil}

Vol. 3, núm. 3., (2019)

Leurys Beatriz Moreira Plaza; Viviana del Rocío Moreira Quiroz; Adriana Carolina Romero Vega; Mayteé Celina Guerrero Cabrera; Fabián Xavier Moreira Rivadeneira; Giancarlo Cristóbal

Cañarte Baque

mujeres post menopaúsicas. Según datos de 1993, la incidencia es de 15 por 100.000 mujeres y la tasa de mortalidad es de 3 por 100.000 mujeres. (Querot, 2017).

- Entre los factores de riesgo más reconocidos, se encuentra la anovulación crónica, la obesidad, la intolerancia a la glucosa y el uso prolongado de estrógenos. La hemorragia post menopaúsica representa el principal síntoma del cáncer de endometrio, admitiéndose que se produce al menos en el $80 \%$ de las mujeres con la enfermedad. Algunas mujeres no manifiestan el síntoma a no ser que se les pregunte, por temor o por desconocimiento del significado de un sangrado después de la menopausia. El pronóstico del cáncer endometrial puede considerarse favorable, pues incluso sin aplicar ninguna medida, la supervivencia oscila entre el $75 \%$ y $80 \%$ a los 5 años.

Actualmente, se considera que la detección precoz de la enfermedad depende de una evaluación adecuada y sin demora de toda hemorragia post menopaúsica. Por ello, es útil y de bajo coste la anamnesis dirigida a la detección de hemorragia post menopaúsica a todas aquellas mujeres climatéricas que acuden al primer nivel de los servicios sanitarios con independencia del motivo de consulta, ya que la sospecha y derivación al nivel especializado para su evaluación adecuada puede beneficiar la supervivencia y calidad de vida de las mujeres afectadas

El cáncer de mama (adenocarcinoma) es una enfermedad maligna en donde la proliferación acelerada, desordenada y no controlada de células pertenecientes a distintos tejidos de la glándula mamaria forman un tumor que invade los tejidos vecinos y metastatiza a órganos distantes del cuerpo. Como otros tumores malignos, el cáncer de mama es consecuencia de alteraciones en la estructura y función de los genes. Los carcinomas de mama suponen más de $90 \%$ de los tumores malignos. Las mamas Cada mama tiene secciones llamadas lóbulos y cada uno de ellos tiene lobulillos más pequeños. Estos terminan en docenas de bulbos diminutos que pueden producir leche. Los lóbulos, lobulillos y bulbos están conectados por tubos delgados llamados conductos. Estos conductos llegan al pezón que está centrado en el área oscura de la piel llamada areola. Los espacios entre lobulillos y los conductos están llenos de grasa. No hay músculos en la mama; los músculos están debajo de cada mama y cubren las costillas. (Brown, 2017). 


\section{Prevención y cuidados ginecológicos de la mujer en edad fértil}

Vol. 3, núm. 3., (2019)

Leurys Beatriz Moreira Plaza; Viviana del Rocío Moreira Quiroz; Adriana Carolina Romero Vega; Mayteé Celina Guerrero Cabrera; Fabián Xavier Moreira Rivadeneira; Giancarlo Cristóbal Cañarte Baque

- Factores de Riesgo: Historia personal de cáncer de mama. Las mujeres que ya tuvieron cáncer de mama enfrentan un mayor riesgo de desarrollar otro cáncer de mama. Historia familiar. El riesgo de una mujer de desarrollar cáncer de mama aumenta si su madre, hermana, hija o dos o más parientes cercanas, como las primas, tuvieron cáncer de mama, sobre todo a una edad joven. Ciertos cambios en los pechos. El haber tenido un diagnóstico de hiperplasia atípica o de carcinoma lobular in situ Alteraciones genéticas Estrógeno. Cuanto más estuvo expuesta una mujer al estrógeno, mayor probabilidad existe que desarrolle cáncer. Sería el caso de mujeres que menstruaron tempranamente y que experimentaron menopausia tardía. La maternidad tardía (mujeres que tuvieron sus hijos después de los 30 años) La radioterapia anterior a los 30 años de edad.

Cáncer de cuello uterino El cáncer de cuello uterino representa un serio problema de salud, dada la alta tasa de mortalidad que existe en torno a esta enfermedad. La gran mayoría de los tumores que se localizan en esta parte del tracto genital tienen un inicio gradual y se detectan fácilmente y son reversibles mediante tratamiento. La realización anual de una toma de muestras de células del cuello (estudio de Papanicolaou o PAP) y la colposcopía -observación del cuello de útero con lentes de aumento desde el inicio de la actividad sexual de las mujeres, han demostrado ser muy efectivos para detectar tumores en forma precoz

- Factores de riesgo Los factores de riesgo del cáncer de cuello uterino son tanto de condición biológica como de carácter psicosocial. Entre los principales factores de riesgo pueden considerarse:

- El que las mujeres no se realicen la prueba de detección periódicamente

- El inicio de las relaciones sexuales a una edad temprana

- El tener parejas sexuales múltiples

- Contraer el virus de inmunodeficiencia humana (VIH) que debilita el sistema inmunológico de la mujer haciéndola más vulnerable a la infección por el Papiloma virus Humano (HPV)

- Presentar una historia familiar de cáncer de cuello uterino

- Encontrarse en edades de entre 30 y 60 años

- Pertenecer a un nivel socio-económico bajo 


\section{Prevención y cuidados ginecológicos de la mujer en edad fértil}

Vol. 3, núm. 3., (2019)

Leurys Beatriz Moreira Plaza; Viviana del Rocío Moreira Quiroz; Adriana Carolina Romero Vega; Mayteé Celina Guerrero Cabrera; Fabián Xavier Moreira Rivadeneira; Giancarlo Cristóbal

Cañarte Baque

\section{- Tener el hábito de fumar.}

Virus de Papiloma Humano (VPH) Es el virus que con mayor frecuencia se transmite por vía sexual. Se encuentra conformado por más de cien tipos de virus, de los cuales cuarenta son propagados sexualmente e infectan al aparato sexual femenino y masculino. Se supone que un 50 $\%$ de los hombres y mujeres sexualmente activos pueden estar infectados con este virus sin tener conocimiento de ello. Esta enfermedad tiene la característica de no presentar síntomas en sus primeras etapas; en el hombre, por ejemplo, puede ser un portador del virus, pero no presentar lesiones a simple vista y, sin embargo, puede estar diseminando el padecimiento entre las mujeres con las que tiene relaciones sexuales sin el uso de protección. (Brown 2017)

- Su método de transmisión es principalmente por contacto sexual, (vaginal o anal) afectando primordialmente los genitales de las mujeres (el cuello del útero, la vagina y el ano) y de los hombres (el pene y el ano). Sus manifestaciones más frecuentes son: las verrugas cutáneas, llamadas también verrugas vulgares y las verrugas en las plantas de los pies. Las lesiones anogenitales se presentan también como las verrugas genitales que son formaciones carnosas con aspecto de coliflor y surgen en las zonas húmedas de los genitales

Información y Seguimiento sobre Métodos Anticonceptivos: Los cuidados médicos ginecológicos preventivos permiten a la mujer controlar su fertilidad referente al número y esparcimiento de sus hijos y disfrutar de su sexualidad sin miedo a los embarazos no deseados y a las enfermedades de transmisión sexual, por lo que adquiere gran importancia la disponibilidad de anticonceptivos.

- Existen diversos métodos anticonceptivos: naturales (coitus interruptus, calendario, temperatura basal,), hormonales (oral, parenteral y post-coital), de barrera (preservativos, diafragma), mecánicos (dispositivo intrauterino -DIU-) y quirúrgicos (ligadura de trompas y vasectomía). Los métodos más utilizados son la anticoncepción hormonal oral y el preservativo.

Climaterio o Menopausia: La menopausia es el episodio final del sangrado menstrual, confirmado por un periodo igual o mayor a seis meses de amenorrea. El climaterio es el periodo de transición de la madurez sexual a la senectud. La edad media de la menopausia se encuentra alrededor de los 


\section{Prevención y cuidados ginecológicos de la mujer en edad fértil}

Vol. 3, núm. 3., (2019)

Leurys Beatriz Moreira Plaza; Viviana del Rocío Moreira Quiroz; Adriana Carolina Romero Vega; Mayteé Celina Guerrero Cabrera; Fabián Xavier Moreira Rivadeneira; Giancarlo Cristóbal Cañarte Baque

50 años. La mujer en esta etapa biológica, demanda atención sanitaria en ocasiones debido en parte a un desconocimiento de las variaciones que se producen en el climaterio y en parte a sintomatología relacionada con la menopausia. (Brown 2017)

- Datos epidemiológicos demuestran que, el $75 \%$ de las mujeres climatéricas tienen ciertos problemas o malestar (específicamente: síntomas vasomotores, síntomas genitourinarios y oscilaciones del humor), aunque solamente el 10-20\% acuden al médico. Se habla de menopausia «normal» cuando no existen síntomas o son leves y, por lo tanto, no necesitan apoyo médico. Se denomina «menopausia patológica» cuando la sintomatología es moderada o grave, obligando a visitar el médico o cuando se produce una menopausia precoz (espontánea, quirúrgica, post tratamiento quimioterápico o radioterápico) antes de los 40 años.

Diversos estudios indican la terapia hormonal sustitutiva (THS) para combatir la sintomatología específica del climaterio y el incremento del riesgo de padecer cardiopatía isquémica y osteoporosis. Sin embargo, el uso de THS no asegura la desaparición del este riesgo y conlleva el riesgo de padecer cáncer de endometrio y cáncer de mama, por lo que su indicación debe ser cuidadosamente analizada y evaluada en cada mujer. (Brown 2017)

\section{Enfoque de los cuidados ginecológicos de la mujer en edad fértil:}

El enfoque que se establece es de carácter preventivo. La detección precoz y el control de las patologías a través de estudios y controles periódicos.

El Programa de prevención del cáncer del cuello uterino obliga a los servicios médicos adheridos al Seguro de Salud, efectuar a todas las mujeres mayores de 25 años que hayan iniciado su vida sexual y hasta los 70 años, un Test de Papanicolaou (PAP). Si éste diera como resultado "normal", repetirlo cada tres (3) años. Si la el PAP diera "anormal", deberá realizarse una colposcopía y un cepillado endocervical. Si estos estudios dieran "normal", repetir el PAP dos (2) veces en un año.

Si estos dos últimos dieron "normal", se continuará con un PAP por año, durante 3 años. De persistir normales los resultados, se hará un control cada 3 años. Si la colposcopía indica patología 


\section{Prevención y cuidados ginecológicos de la mujer en edad fértil}

Vol. 3, núm. 3., (2019)

Leurys Beatriz Moreira Plaza; Viviana del Rocío Moreira Quiroz; Adriana Carolina Romero Vega; Mayteé Celina Guerrero Cabrera; Fabián Xavier Moreira Rivadeneira; Giancarlo Cristóbal

Cañarte Baque

o "anormal", se debe realizar una biopsia dirigida por colposcopía; el médico/a indicará el tratamiento adecuado y oportuno. Si la biopsia es "negativa", se realizará un control anual con PAP y colposcopía. Si estos controles fueran "normales", se continuará con el control cada 3 años.

El Programa de prevención del cáncer de mama funda su acción en la detección precoz del cáncer. Hace hincapié en que se debe promover y enseñar a efectuar el autoexamen mamario -incluyendo la palpación de la axila- con planes de educación, folletos ilustrativos y otras estrategias de comunicación. Establece niveles diferentes de riesgo para las mujeres con antecedentes familiares de cáncer de mama y aquella población en riesgo standard. Para las mujeres por debajo de los 40 años con una fuerte historia familiar de la enfermedad, se recomienda la realización de una mamografía a los 36 años y una mamografía anual, a partir de los 40 .

Prevención de cáncer de cuello uterino Entre las medidas preventivas para el diagnóstico oportuno se cuenta con una herramienta que es la Prueba del Papanicolaou (PAP). Ésta debe realizarse a toda mujer con vida sexual activa.25 Prueba De Papanicolaou: Esta prueba toma el nombre del investigador que observó por primera vez las células del cérvix y útero en un frotis de secreciones vaginales, en la cual se detectó células anormales o atípicas en etapas tempranas del cáncer de cuello uterino. La prueba consiste en realizar los siguientes pasos:

$1^{\circ}$.- Por medio de un espéculo (instrumento que sirve para abrir la vagina) el médico o la médica observa con atención el cuello del útero y raspa con un cepillito la superficie del mismo para tomar una muestra de los tejidos. No duele ni causa molestias.

$2^{\circ}$.- Las células se colocan sobre una lámina de vidrio y se envían al laboratorio para que un patólogo las analice. Existe una manera de informar los resultados cuya clasificación general es como la siguiente:

- Clase I: Negativo o normal. Es cuando las células son normales y no hubo cambio en ellas.

- Clase II: irregular o anormal. Cuando las células son ligeramente anormales: esto puede ser debido a una pequeña inflamación de los tejidos del cuello de útero. Por lo general no 


\section{Prevención y cuidados ginecológicos de la mujer en edad fértil}

Vol. 3, núm. 3., (2019)

Leurys Beatriz Moreira Plaza; Viviana del Rocío Moreira Quiroz; Adriana Carolina Romero Vega; Mayteé Celina Guerrero Cabrera; Fabián Xavier Moreira Rivadeneira; Giancarlo Cristóbal Cañarte Baque

se indica tratamiento pues muchas de las células vuelven con el tiempo a la normalidad sin tratamiento médico. En general el/la médica recomienda repetir el PAP a los 3 ó 6 meses. También podrá pedir una biopsia para confirmar los resultados del PAP.

- Clase III: sospechoso o displasia. No es cáncer. Es cuando las células cambiaron y hay que continuar investigando para determinar el grado de cambio anormal. Su evolución es impredecible: a veces mejora sin tratamiento o continúa, por eso hay que controlarla con pruebas regulares. Las mujeres entre 25 y 35 años de edad corren un mayor riesgo de tenerla.

- Clase IV y $V$ : Probabilidad de carcinoma in situ o de cáncer invasor. Aparecen con resultado positivo en el PAP lo que indica una probabilidad de cáncer. Sólo una biopsia puede determinar de qué células se trata. En cualquiera de los casos, requerirán tratamiento.

\section{Conclusiones.}

La salud de la mujer ofrece un marco extremadamente interesante para la realización de medicina preventiva. Dado que durante su vida a partir de la adolescencia hasta los años posteriores al climaterio mantendrá un estrecho contacto con su obstetra o ginecólogo estableciéndose habitualmente una relación estrecha de confianza y duradera. El Examen Ginecológico es una forma más efectiva para detectar enfermedades patológicas que pueden encontrarse en los órganos reproductivos (vulva, vagina, cuello del útero, endometrio, útero, trompas, ovarios) y mamas, y así poder prevenir y curar estas enfermedades.

Este examen es recomendado en la mujer en todas sus edades; pero, especialmente las que va desde los 15 hasta los 44 años y se recomienda a que toda mujer que haya iniciado su vida sexual se lo realice por lo menos una vez al año, ya que sirve para la identificar, diagnosticar, prevenir y tratar enfermedades de orden patológico, entre otras de mayor incidencia a nivel mundial, esta interacción de orden preventiva en las diferentes etapas del ciclo vital de la mujer brinda la 


\section{Prevención y cuidados ginecológicos de la mujer en edad fértil}

Vol. 3, núm. 3., (2019)

Leurys Beatriz Moreira Plaza; Viviana del Rocío Moreira Quiroz; Adriana Carolina Romero Vega; Mayteé Celina Guerrero Cabrera; Fabián Xavier Moreira Rivadeneira; Giancarlo Cristóbal

Cañarte Baque

extraordinaria oportunidad de poder interactuar en forma proactiva en su salud en distintos procesos durante distintas edades de la vida femenina.

Puede ser que se llegue a considerar una consulta de endocrinología ginecológica orientada a investigar y manejar las alteraciones del ciclo menstrual y del equilibrio hormonal, con especial énfasis en las consecuencias que éstas pueden tener para la salud de la mujer como el sobrepeso y los problemas cardiovasculares.

Además de esta primera consulta destinada a "romper el hielo", la mujer tiene la obligación de asistir al especialista en cuanto haya iniciado su vida sexual; y si no ha tenido su iniciación hasta los 20 años, ésta es la edad más adecuada para empezar los controles periódicos. Lo ideal es visitar al ginecólogo una vez al año en caso de presentar un cuadro normal; y si se detecta algún inconveniente, asistir todas las veces que el médico lo indique.

En términos generales, los cuidados ginecológicos le permiten a la mujer en edad fértil llevar un control de rutina, el cual le permitirá hacer énfasis en la prevención y el autocuidado; en la consulta pre concepcional, en la identificación de factores de riesgo y la preparación para el embarazo. Asimismo, le facilitará el hecho de desarrollar un control prenatal especializado, durante su embarazo, el cual le permitirá alcanzar los mejores resultados para ella y su hijo o hija.

De igual forma, este tipo de cuidados preventivos le permiten a la mujer concebir una consulta de patología cervical, la cual se especializa en el diagnóstico y tratamiento de cualquier anormalidad que se detecte en el cuello del útero mediante la citología, en especial las relacionadas con el virus del papiloma humano; durante este proceso el doctor brinda apoyo integral, diagnóstico y tratamiento a la pareja que tiene dificultades en lograr un embarazo.

Finalmente, los cuidados ginecológicos brindan a la mujer la posibilidad de implementar el manejo adecuado de los síntomas climatéricos y en la prevención de complicaciones como lo es el cáncer de ovarios, de mamas, o cualquier patología de tipo oncológico. En pocas palabras, sea cual sea el momento de la vida en que se acuda al especialista, en cada consulta de ginecología se da inicialmente una conversación que tiene como objetivo conocer a la mujer y sus necesidades 


\section{Prevención y cuidados ginecológicos de la mujer en edad fértil}

Vol. 3, núm. 3., (2019)

Leurys Beatriz Moreira Plaza; Viviana del Rocío Moreira Quiroz; Adriana Carolina Romero

Vega; Mayteé Celina Guerrero Cabrera; Fabián Xavier Moreira Rivadeneira; Giancarlo Cristóbal Cañarte Baque

individuales, lo cual le permitirá a la misma, desarrollar un ambiente de confianza y expresar todas sus dudas y temores sin miedo a ser juzgada o ignorada, lográndose con ello, prevenir anomalías que podrían ser mortales a futuro.

\section{Bibliografía.}

Arias, F. (2010). Paradigmas de la Investigación Cientifica. España: Editorial: Luces.

Brown, V. (2017). Epidemiolog Oncolgy Cancer. Ther Journal Oncology, 12.

Calls, B. (2016). Lesiones Oncológicas. Revista Española de Obstetricia y Medicina Interna de la Universidad Computense de Madrid, España., 19.

Cett, P. (2016). Cuidados Ginecológicos una herramienta de orden Sanitario. Revista de Atención Asistencial y Sanitaria de Guatemala., 14.

Dávila, N. (2012). Paradigmas de la Investigación Científica. Pereire, Colombia: Editorial: Las Brisas.

Pallos, G. (2016). Programas de Atención Primaria de Salud en Obstetricia. Revista: Salud l de la Universidad La Rioja.Colombia., 21.

Perroni, C. (2016). Incidencia de Cáncer de Mamas en Mujeres en Edad Fértil. Revista de Obstetricia de la Universidad Nacional de Perú., 19.

Querot, Y. (2017). Edades fértiles en la Mujer. Revista The Journal Medic Cáncer, 23.

Rawy, E. (2015). Estudios Ginecológicos en la Atención Primaria de Salud. Revista Médica La Habana, Cuba., 12.

Riantt, T. (2017). Controles Ginecológicos de la Mujer en edad Fecunda. Revista Médica de la Escuela de Obstetricia de la Universidad Central de Venezuela. UCV, Caracas. , 11.

$$
\text { (c) (1) () () (2) }
$$

\section{RECONOCIMIENTO-NOCOMERCIAL-COMPARTIRIGUAL \\ CC BY-NC-SA}

ESTA LICENCIA PERMITE A OTROS ENTREMEZCLAR, AJUSTAR Y CONSTRUIR A PARTIR DE SU OBRA CON FINES NO COMERCIALES, SIEMPRE Y CUANDO LE RECONOZCAN LA AUTORÍA Y SUS NUEVAS CREACIONES ESTÉN BAJO UNA LICENCIA CON LOS MISMOS TÉRMINOS. 